\title{
Human placental vasculature imaging using an LED-based photoacoustic/ ultrasound imaging system
}

Efthymios Maneas, Wenfeng Xia, Mithun Kuniyil Ajith Singh, Naoto Sato, Toshitaka Agano, et al.

Efthymios Maneas, Wenfeng Xia, Mithun Kuniyil Ajith Singh, Naoto Sato, Toshitaka Agano, Sebastien Ourselin, Simeon J. West, Anna L. David, Tom Vercauteren, Adrien E. Desjardins, "Human placental vasculature imaging using an LED-based photoacoustic/ultrasound imaging system

," Proc. SPIE 10494, Photons Plus Ultrasound: Imaging and Sensing 2018, 104940Y (19 February 2018); doi: 10.1117/12.2288995

SPIE. Event: SPIE BiOS, 2018, San Francisco, California, United States 


\title{
Human placental vasculature imaging using an LED-based photoacoustic/ultrasound imaging system
}

\author{
Efthymios Maneas ${ }^{\mathrm{a}, \mathrm{b}, *}$, Wenfeng Xia ${ }^{\mathrm{a}, \mathrm{b}}$, Mithun Kuniyil Ajith Singh ${ }^{\mathrm{c}}$, Naoto Sato ${ }^{\mathrm{d}}$, Toshitaka \\ Agano $^{\mathrm{d}}$, Sebastien Ourselin ${ }^{\mathrm{a}, \mathrm{b}}$, Simeon J. West ${ }^{\mathrm{e}}$, Anna L. David ${ }^{\mathrm{a}, \mathrm{g}, \mathrm{g}}$, Tom Vercauteren ${ }^{\mathrm{a}, \mathrm{b}}$, \\ Adrien E. Desjardins ${ }^{\mathrm{a}, \mathrm{b}}$ \\ ${ }^{a}$ Wellcome / EPSRC Centre for Interventional and Surgical Sciences, University College London, \\ Charles Bell House, 67-73 Riding House Street, London W1W 7EJ, United Kingdom; \\ ${ }^{\mathrm{b}}$ Department of Medical Physics and Biomedical Engineering, University College London, Gower \\ Street, London WC1E 6BT, United Kingdom; \\ ${ }^{\mathrm{c}}$ Research and Business Development Division, PreXion Corporation, Cambridge Innovation Center, \\ Stationsplein 45, A4.004, 3013 AK Rotterdam, The Netherlands; \\ ${ }^{\mathrm{d}}$ Research and Development Division 1, PreXion Corporation, 1-14-1 Kandasudacho, Chiyoda-ku, \\ 101-0041, Tokyo, Japan; \\ ${ }^{\mathrm{e}}$ Department of Anaesthesia, University College Hospital, Main Theatres, Maple Bridge Link \\ Corridor, Podium 3, 235 Euston Road, London NW1 2BU, United Kingdom; \\ ${ }^{\mathrm{f}}$ Institute for Women's Health, University College London, 86-96 Chenies Mews, London WC1E \\ $6 \mathrm{HX}$, United Kingdom; \\ ${ }^{\mathrm{g}}$ Department of Development and Regeneration, KU Leuven (Katholieke Universiteit), Belgium; \\ *efthymios.maneas.14@ucl.ac.uk
}

\begin{abstract}
Minimally invasive fetal interventions, such as those used for therapy of twin-to-twin transfusion syndrome (TTTS), require accurate image guidance to optimise patient outcomes. Currently, TTTS can be treated fetoscopically by identifying anastomosing vessels on the chorionic (fetal) placental surface, and then performing photocoagulation. Incomplete photocoagulation increases the risk of procedure failure. Photoacoustic imaging can provide contrast for both haemoglobin concentration and oxygenation, and in this study, it was hypothesised that it can resolve chorionic placental vessels. We imaged a term human placenta that was collected after caesarean section delivery using a photoacoustic/ultrasound system (AcousticX) that included light emitting diode (LED) arrays for excitation light and a linear-array ultrasound imaging probe. Two-dimensional (2D) co-registered photoacoustic and B-mode pulse-echo ultrasound images were acquired and displayed in real-time. Translation of the imaging probe enabled 3D imaging. This feasibility study demonstrated that photoacoustic imaging can be used to visualise chorionic placental vasculature, and that it has strong potential to guide minimally invasive fetal interventions.
\end{abstract}

Keywords: Photoacoustic imaging, ultrasound imaging, LED, human placental vasculature

\section{INTRODUCTION}

Minimally invasive fetal interventions, such as those used for treatment of twin-to-twin transfusion syndrome (TTTS), require accurate image guidance to optimise patient outcomes ${ }^{1}$. TTTS occurs from an imbalance of blood flow across inter-twin vascular connections (anastomoses) in the placenta, and it is associated with high morbidity and mortality risks if left untreated ${ }^{2}$. Currently, TTTS can be treated by identifying anastomosing vessels on the chorionic (fetal) placental surface using a fetoscope, and then performing laser photocoagulation using an optical fibre inserted through the working channel ${ }^{3,4}$. Incomplete photocoagulation due to inadequate visualisation of vessels increases the risk of procedure failure and perinatal death ${ }^{5}$. In current clinical practice for TTTS procedures, in vivo imaging of the placenta is

Photons Plus Ultrasound: Imaging and Sensing 2018, edited by Alexander A. Oraevsky, Lihong V. Wang, Proc. of SPIE Vol. 10494, 104940Y · @ 2018 SPIE · CCC code: 0277-786X/18/\$18 · doi: 10.1117/12.2288995 
performed using white light fetoscopy and external B-mode ultrasound (US) imaging. Both of these modalities can provide insufficient contrast to visualise small anastomosing vessels beneath the chorionic placental surface ${ }^{6}$. Photoacoustic imaging (PA) can provide rich optical absorption contrast for haemoglobin concentration and oxygenation $^{7-9}$. Previous studies demonstrated that PA signals from placental vasculature can be detected with an external clinical ultrasound probe $e^{10,11}$. In this study, a post-partum term human placenta was imaged with a PA/US imaging system that included PA excitation light by light emitting diode (LED) arrays.

\section{MATERIALS AND METHODS}

A real-time PA/US system ${ }^{12}$ (AcousticX, PreXion Corporation, Tokyo, Japan), which comprised LED arrays for excitation light delivery and a clinical linear-array ultrasound imaging probe $(9 \mathrm{MHz}$ central frequency, 128-element, $300 \mu \mathrm{m}$ pitch, PreXion Corporation) for ultrasound reception, was used to image placental vasculature. Excitation light at $850 \mathrm{~nm}(200 \mu \mathrm{J}$ nominal pulse energy per array; $4 \mathrm{KHz}$ pulse repetition frequency) was provided using two LED arrays mounted in each side of the ultrasound probe, over an illumination area of $50 \mathrm{~mm} \times 7 \mathrm{~mm}$ on the surface of the placenta. Raw acoustic signals from 128 channels were recorded and digitised simultaneously at $40 \mathrm{MS} / \mathrm{s}$; real time 2D co-registered photoacoustic and B-mode pulse-echo ultrasound images were reconstructed and displayed at a frame rate of $6.25 \mathrm{~Hz}$, with signal averaging performed over 384 signal acquisitions. Translation of the imaging probe with a linear motorised stage supplied with the system (OSMS20-85(X), OptoSigma Corporation, California, USA) enabled 3D imaging. The translated distance during a 3D image volume acquisition was $40 \mathrm{~mm}$. Offline reconstruction of the recorded PA signals was performed using a frequency-domain algorithm implemented with the k-Wave Matlab toolbox ${ }^{13}$.

To investigate the feasibility of the system to visualise superficial and subsurface placental vessels on the fetal chorionic placenta, a normal term placenta was collected with written informed consent after a caesarean section delivery at University College London Hospital. Our local committees on the Ethics of Human Research approved the study (14/LO/0863). The umbilical cord was clamped immediately after the delivery to preserve the blood inside the vessels. The placenta was initially placed in a plastic container and subsequently it was coated with ultrasound gel for acoustic coupling and covered with cling film. The container was filled with water at room temperature for acoustic coupling and for free translation of the imaging probe (Figure 1). Several highly vascularised locations on the surface of the chorionic fetal side were imaged (Figures 2 and 3).

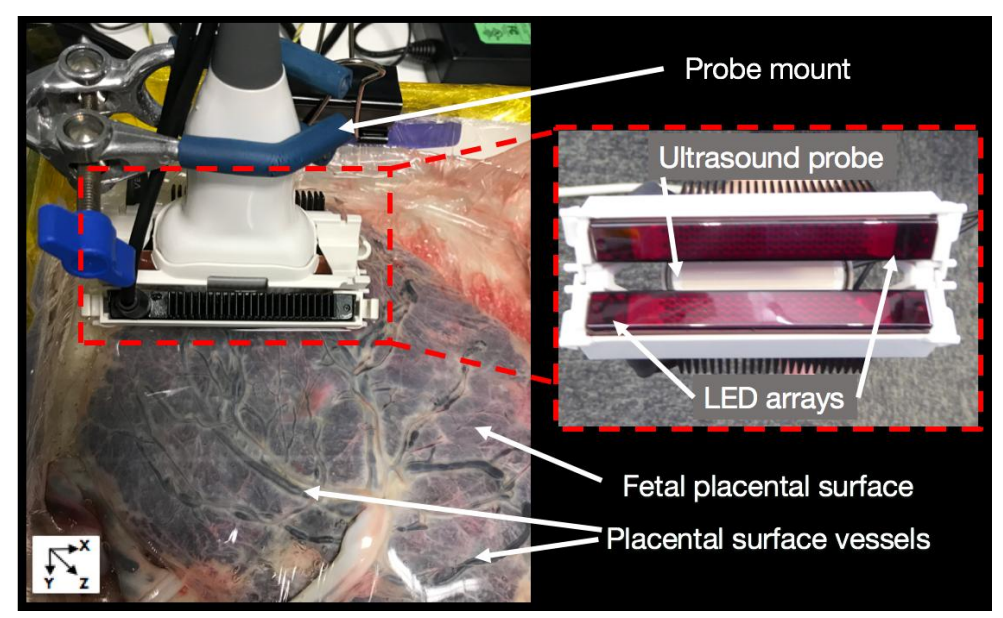

Figure 1. Experimental setup to image a human placenta. A clamp was used to mount the ultrasound probe with the light emitting diode (LED) arrays to the linear motorised stage. The placenta was coated with ultrasound gel, covered with cling film, and placed inside a water-filled container. 


\section{RESULTS}

Individual PA and US images of a term human chorionic placental vasculature that were acquired in real-time are presented in Figure 2. With PA imaging, superficial blood vessels and a subsurface structure were visible to a depth of approximately $5 \mathrm{~mm}$ from the placental chorionic fetal surface. Some of these vascular structures were not apparent in US images. With US imaging, a large blood vessel located at a depth of approximately $7 \mathrm{~mm}$ could be identified, but this vessel was not apparent with PA imaging. Figure 3 shows a photograph of the area that was imaged and the corresponding top and side maximum intensity projections (MIPs) of the reconstructed 3D photoacoustic signals. Several superficial branching vessels were clearly resolved. In the top view MIP PA image, high intensity PA signals appeared to originate from vascular structures that were not visible in the photograph.
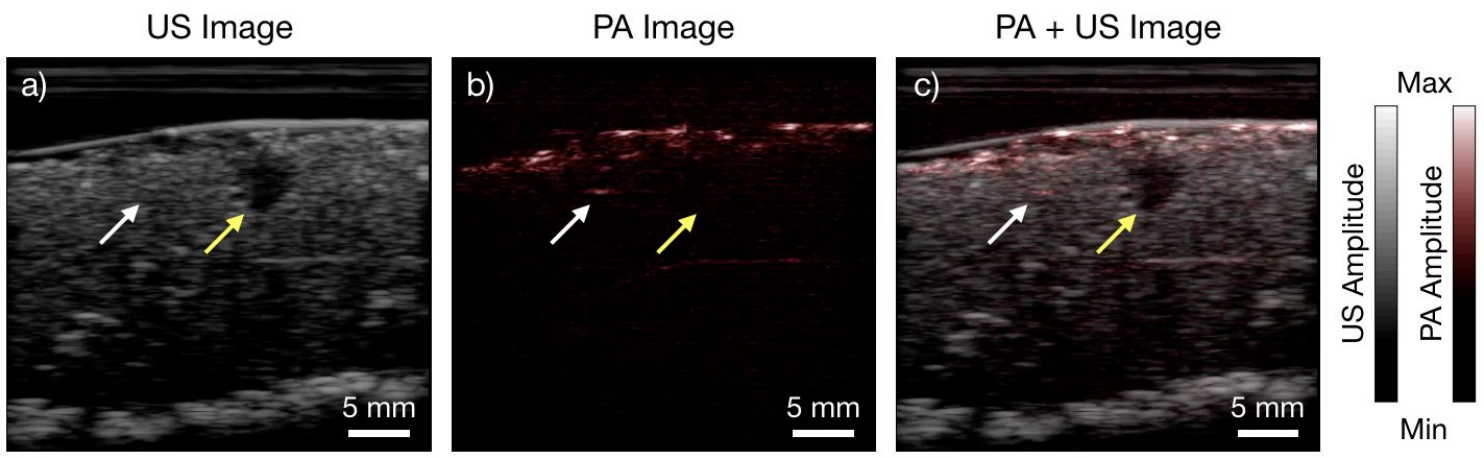

Figure 2. Single frames of ultrasound (US), photoacoustic (PA), and merged US and PA images acquired from a human placenta, at one location $(\mathrm{a}-\mathrm{c})$. A large blood vessel (yellow arrow) that is visible in the US image was not visible in the PA image. A subsurface structure was visible with PA imaging (white arrow). All the images are displayed on logarithmic scales.
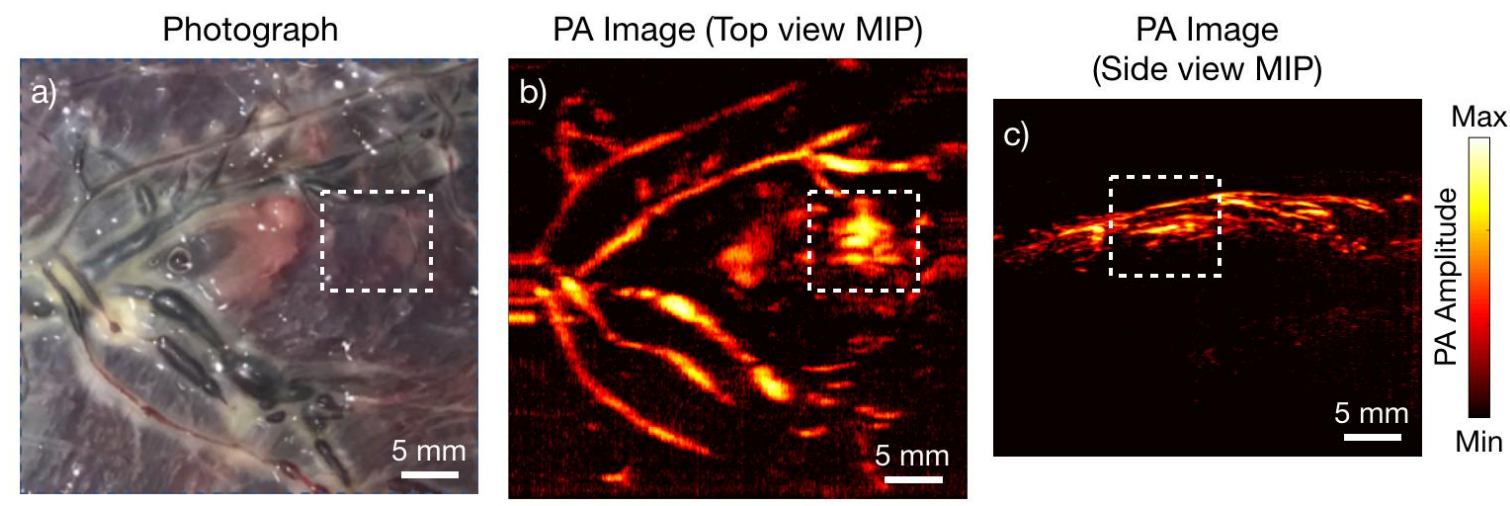

Figure 3. Photograph (a) and photoacoustic (PA) images (b,c) of a portion of the human placenta. Superficial branching blood vessels are apparent in both the photograph and the PA images. High intensity PA signals (dashed squared box) that are not visible in the photograph might be attributable to subsurface vascular structures. The PA images are displayed on a logarithmic scale as maximum intensity projections (MIPs) of the reconstructed 3D photoacoustic image volume.

\section{DISCUSSION AND CONCLUSIONS}

This preliminary study demonstrated the feasibility of real-time LED-based PA/US imaging of the human placenta. LED arrays adjacent to the probe for delivering excitation light allowed for a compact console, and with their high repetition 
rate, averaging across consecutive PA image frames could be performed whilst maintaining a real-time display. The depth range of anastamosing vessels in TTTS is not known; however, it may well be larger than the PA imaging depth of the system used in this study. To increase PA imaging depths, next-generation systems could include the use of coded excitation to increase the signal-to-noise ratio of the generated PA signals ${ }^{14}$ or more powerful LED sources ${ }^{15}$.

For clinical translation of PA imaging to guide TTTS, it will likely be necessary to develop miniature probes that have sufficiently small lateral dimensions to allow for delivery through the working channel of a fetoscope. These probes would likely deliver excitation light at the distal end; ultrasound reception could either also be at the distal end or it could be performed by an US imaging probe external to the mother ${ }^{10,11,16-18}$. Multispectral PA could be used to obtain information about oxygen saturation in vessels, providing insights into the pathogenesis of TTTS. Post-partum vasoconstriction may have limited contrast for deep vessels with both US and PA imaging. Therefore, imaging of a perfused placenta could potentially improve visualisation of vessels at larger depths. By visualising superficial and subsurface chorionic vascular structures in the placenta, PA imaging may be useful to confirm successful laser ablation of anastamosing vessels during TTTS fetoscopic therapy and thereby to improve treatment outcomes.

\section{ACKNOWLEDGEMENTS}

This work was supported by an Innovative Engineering for Health award by the Wellcome Trust [WT101957] and the Engineering and Physical Sciences Research Council (EPSRC) [NS/A000027/1], by a Starting Grant from the European Research Council [ERC-2012-StG, Proposal 310970 MOPHIM], by an EPSRC First Grant [EP/J010952/1], by a Wellcome / EPSRC Centre award [203145Z/16/Z \& NS/A000050/1], by a Royal Society Research Grant [RG160569] and by the UCL EPSRC Centre for Doctoral Training in Medical Imaging [EP/L016478/1]. A.L.D. is supported by the UCL/UCLH NIHR Comprehensive Biomedical Research Centre.

\section{REFERENCES}

[1] Pratt, R., Deprest, J., Vercauteren, T., Ourselin, S. and David, A.L., "Computer-assisted surgical planning and intraoperative guidance in fetal surgery: a systematic review," Prenat. Diagn. 35(12), 1159-1166 (2015).

[2] Lewi, L., Deprest, J. and Hecher, K., "The vascular anastomoses in monochorionic twin pregnancies and their clinical consequences," J. Obstet. Gynecol. 208(1), 19-30 (2013).

[3] Deprest, J.A., Flake, A.W., Gratacos, E., Ville, Y., Hecher, K., Nicolaides, K., Johnson, M.P., Luks, F.I., Adzick, N.S. and Harrison, M.R., "The making of fetal surgery," Prenat. Diagn. 30(7), 653-667 (2010).

[4] Slaghekke, F., Lopriore, E., Lewi, L., Middeldorp, J.M., van Zwet, E.W., Weingertner, A.S., Klumper, F.J., DeKoninck, P., Devlieger, R., Kilby, M.D. and Rustico, M.A., "Fetoscopic laser coagulation of the vascular equator versus selective coagulation for twin-to-twin transfusion syndrome: an open-label randomised controlled trial," Lancet 383(9935), 2144-2151 (2014).

[5] Lewi, L., Jani, J., Cannie, M., Robyr, R., Ville, Y., Hecher, K., Gratacos, E., Vandecruys, H., Vandecaveye, V., Dymarkowski, S. and Deprest, J., "Intertwin anastomoses in monochorionic placentas after fetoscopic laser coagulation for twin-to-twin transfusion syndrome: is there more than meets the eye?" J. Obstet. Gynecol. 194(3), 790-795 (2006).

[6] Guiot, C., Gaglioti, P., Oberto, M., Piccoli, E., Rosato, R. and Todros, T., "Is three-dimensional power Doppler ultrasound useful in the assessment of placental perfusion in normal and growth-restricted pregnancies?" Ultrasound Obstet. Gynecol. 31(2), 171-176 (2008).

[7] Beard, P., "Biomedical Photoacoustic Imaging," Interface Focus 1(4), 602-631 (2011).

[8] Wang, L.V., and Hu, S., "Photoacoustic tomography: in vivo imaging from organelles to organs," Science 335(6075), 1458-1462 (2012).

[9] Ntziachristos, V., and Razansky, D., "Molecular imaging by means of multispectral optoacoustic tomography (MSOT)," Chem. Rev. 110(5), 2783-94 (2010).

[10] Xia, W., Maneas, E., Nikitichev, D.I., Mosse, C.A., dos Santos, G.S., Vercauteren, T., David, A.L., Deprest, J., Ourselin, S., Beard, P.C. and Desjardins, A.E., "Interventional photoacoustic imaging of the human placenta with ultrasonic tracking for minimally invasive fetal surgeries," Med. Image Comput. Comput. Assist. Interv., 371-378 (2015). 
[11] Xia, W., Nikitichev, D.I., Mari, J.M., West, S.J., Pratt, R., David, A.L., Ourselin, S., Beard, P.C. and Desjardins, A.E., "Performance characteristics of an interventional multispectral photoacoustic imaging system for guiding minimally invasive procedures," J Biomed. Opt. 20(8), 086005 (2015).

[12] Hariri, A., Lemaster, J., Wang, J., Jeevarathinam, A.S., Chao, D.L. and Jokerst, J.V., "The characterization of an economic and portable LED-based photoacoustic imaging system to facilitate molecular imaging," Photoacoustics 9, 10-20 (2018).

[13] Treeby, B.E., and Cox, B.T., "k-Wave: MATLAB toolbox for the simulation and reconstruction of photoacoustic wave fields," J. Biomed. Opt. 15(2), 021314 (2010).

[14] Xia, W., Ginsberg, Y., West, S.J., Nikitichev, D.I., Ourselin, S., David, A.L. and Desjardins, A.E., “Coded excitation ultrasonic needle tracking: An in vivo study," Med. Phys. 43(7), 4065-4073 (2016).

[15] Allen, T.J., and Beard, P.C., "High power visible light emitting diodes as pulsed excitation sources for biomedical photoacoustics," Biomed. Opt. Express 7(4), 1260-1270 (2016).

[16] Mari, J.M., Xia, W., West, S.J. and Desjardins, A.E., "Interventional multispectral photoacoustic imaging with a clinical ultrasound probe for discriminating nerves and tendons: an ex vivo pilot study," J. Biomed. Opt. 20(11), 110503 (2015).

[17] Bell, M.A.L., Kuo, N.P., Song, D.Y., Kang, J.U. and Boctor, E.M., "In vivo visualization of prostate brachytherapy seeds with photoacoustic imaging," J. Biomed. Opt. 19(12), 126011 (2014).

[18] Singh, M.K.A., Parameshwarappa, V., Hendriksen, E., Steenbergen, W. and Manohar, S., "Photoacousticguided focused ultrasound for accurate visualization of brachytherapy seeds with the photoacoustic needle," J. Biomed. Opt. 21(12), 120501 (2016). 